\title{
Exosomes Derived from Stem Cells from the Apical Papilla Promote Dentine-Pulp Complex Regeneration by Inducing Specific Dentinogenesis
}

\author{
Xueying Zhuang $\mathbb{D}$, ${ }^{1,2}$ Lingli Ji $\mathbb{C D}^{1,2}$ Huan Jiang $\mathbb{D}^{1,2}$ Yao Liu $\mathbb{D}^{1,2}$ Xuemei Liu, ${ }^{1,2}$ Jing Bi, ${ }^{1,2}$ \\ Weidong Zhao $\mathbb{D},{ }^{3}$ Zhenjiang Ding $\mathbb{D}^{1,2}$ and $X u$ Chen $\mathbb{D}^{1,2}$ \\ ${ }^{1}$ Department of Pediatric Dentistry, School and Hospital of Stomatology, China Medical University, Shenyang 110002, China \\ ${ }^{2}$ Liaoning Provincial Key Laboratory of Oral Diseases, Shenyang 110002, China \\ ${ }^{3}$ Department of Developmental Cell Biology, School of Life Sciences, China Medical University, Shenyang 110122, China \\ Correspondence should be addressed to Xu Chen; chenxu@cmu.edu.cn
}

Received 22 January 2020; Revised 26 March 2020; Accepted 12 May 2020; Published 27 May 2020

Academic Editor: Gerald A. Colvin

Copyright () 2020 Xueying Zhuang et al. This is an open access article distributed under the Creative Commons Attribution License, which permits unrestricted use, distribution, and reproduction in any medium, provided the original work is properly cited.

\begin{abstract}
Regenerative endodontic procedures (REPs) are a new option for the treatment of dental pulp or periapical diseases in permanent teeth with open apices. Histologically, the new tissues formed in the root canal after REPs are mainly cementum- or bone-like mineralised tissues, but not the real dentine-pulp complex. Therefore, how to promote dentine-pulp complex regeneration and improve the clinical effects of REPs has become a prominent research topic. Stem cells from apical papilla (SCAP) are derived from the dental papilla that can differentiate into primary odontoblasts and dental pulp cells that produce root dentine and dental pulp. Exosomes are the key regulator for the paracrine activity of stem cells and can influence the function of recipient cells. In this study, SCAP-derived exosomes (SCAP-Exo) were introduced into the root fragment containing bone marrow mesenchymal stem cells (BMMSCs) and transplanted subcutaneously into immunodeficient mice. We observed that dental pulp-like tissues were present and the newly formed dentine was deposited onto the existing dentine in the root canal. Afterwards, the effects of SCAP-Exo on the dentinogenesis of BMMSCs were elucidated in vitro. We found that the gene and protein expression of dentine sialophosphoprotein and mineralised nodule formation in BMMSCs treated with SCAP-Exo were significantly increased. In summary, SCAP-Exo were endocytosed by BMMSCs and obviously improved their specific dentinogenesis. The use of exosomes derived from dental stem cells could comprise a potential therapeutic approach for dentine-pulp complex regeneration in REPs.
\end{abstract}

\section{Introduction}

The treatment of dental pulp or periapical diseases in permanent teeth with open apices presents challenges to clinicians. The traditional method of apexification is restricted by the incomplete root formation with the open apex and short root. Regenerative endodontic procedures (REPs), a new treatment option for immature teeth with necrotic pulp and/or periapical periodontitis, are a biological method based on the concept of the triad of tissue engineering [1]. The tissue regeneration in REPs is mainly due to the cell-homing effect, which is directed by autogenous dental stem cells recruited into the empty root canal, such as residual dental pulp stem cells, stem cells from apical papilla (SCAP), periodontal ligament stem cells, and bone marrow mesenchymal stem cells (BMMSCs) from the jaw bone [2, 3]. REPs allow for the elimination of symptoms and bony healing and result in continued root development with increased root wall thickness and increased root length. However, our previous study showed that free cellular cementum-like tissues and bone-like mineralised structures are formed after REPs in the teeth of beagles with periapical periodontitis [4]. Studies have also indicated that the newly formed tissues in the canal after REPs are not real dentine-pulp complex but 
rather heterogeneous tissues including connective tissue, cementum-like tissue, and bone-like tissue [5, 6]. Further, the survival of dental stem cells in cases of pulp necrosis and apical periodontitis is not sufficient, and BMMSCs from the jaw bone might be the main stem cells that are homing to the root canal during REPs [7]. Therefore, how to promote dentine-pulp complex regeneration and improve the clinical effects of REPs have become a prominent research topic.

Following the development of tissue engineering, recent evidence has indicated that the tissue regeneration based on mesenchymal stem cells (MSCs) is mainly dependent on paracrine effects such as the secretion of trophic factors, cytokines, and extracellular vesicles [8]. Exosomes derived from mesenchymal stem cells (MSC-Exo) are extracellular microvesicles with a cup-shaped double-membrane structure and a size of $30-150 \mathrm{~nm}$ in diameter [9, 10]. Further, MSC-Exo contain growth factors; are rich in bioactive lipids, proteins, mRNAs, and regulatory miRNA; play important roles in cell-cell communication; and can influence the function of recipient cells $[11,12]$. MSC-Exo serve as paracrine or autocrine mediators that regulate tissue regeneration. To date, MSC-Exo have been applied extensively to tissue regeneration and restoration modalities such as alleviating traumatic brain injury, enhancing cardiac repair, protecting the kidney from acute ischemia-reperfusion injury, and promoting muscle regeneration, among other applications [13-16].

SCAP play a critical role in tooth development and pulp regeneration in permanent teeth with open apices. SCAP can differentiate into primary odontoblasts and dental pulp cells that produce root dentine and dental pulp, thus serving as a promising stem cell source for dental pulp regeneration. Moreover, exosomes derived from stem cells from the apical papilla (SCAP-Exo) might carry the specific biological information of SCAP. Thus, we hypothesized that SCAP-Exo administration during REPs might enhance dentinogenesis and improve dentine-pulp complex regeneration. In this study, we evaluated the potential of SCAP-Exo to facilitate dentine-pulp complex regeneration, as well as the possible regulatory effects of SCAP-Exo on the biological function of BMMSCs, especially with respect to dentinogenesis capacity.

\section{Materials and Methods}

2.1. SCAP Isolation and Identification. Human impacted third molar with immature roots were collected from each healthy patient (12-15 years old) in the Dental Clinics at the School of Stomatology affiliated with China Medical University. The study was approved by the Ethics Committee of the School of Stomatology, China Medical University (201508). The apical papilla was gently removed from the tooth, minced, and digested in a solution of $2 \mathrm{mg} / \mathrm{mL}$ collagenase type I (Worthington, USA) and $4 \mathrm{mg} / \mathrm{mL}$ dispase II (Mannheim, Germany). Single-cell suspensions were seeded in $10 \mathrm{~cm}$ culture dishes and cultured with an alpha minimum essential medium ( $\alpha$-MEM, HyClone, USA) supplemented with $15 \%$ fetal bovine serum (FBS, MRC BRL), $2 \mathrm{mM}$ L-glutamine (Biosource/Invitrogen, USA), $100 \mathrm{U} / \mathrm{mL}$ penicillin-streptomycin (HyClone), and $0.1 \mathrm{mM} \mathrm{L-ascorbic}$ acid 2-phosphate (WAKO, Japan) and maintained in 5\%
$\mathrm{CO}_{2}$ at $37^{\circ} \mathrm{C}$. SCAP were identified by flow cytometry using anti-CD29, CD44, CD105, CD146, CD34, and CD45 antibodies (Abcam, USA). The multipotent differentiation potentials of osteogenesis and adipogenesis of SCAP were evaluated after osteogenic and adipogenic induction for 4 weeks. Alizarin red $\mathrm{S}$ and Oil red $\mathrm{O}$ staining were used to detect the formation of mineralised nodules and lipid droplets. SCAP from the third passage were used in the experiment.

2.2. BMMSC Isolation and Identification. Six to eight weeks Wistar rats (purchased from Vitaliver, Beijing, China) were selected and anesthetized with $2 \%$ pentobarbital sodium. The femurs and tibia of the hind limbs were separated to expose the bone marrow cavity. Then, $100 \mathrm{U} / \mathrm{mL}$ penicillinstreptomycin and 2\% BSA (Meilun, China) were used to rush out the bone marrow. Single-cell suspensions were seeded in $10 \mathrm{~cm}$ culture dishes and cultured with the conventional medium in $5 \% \mathrm{CO}_{2}$ at $37^{\circ} \mathrm{C}$. The multipotent differentiation potentials of osteogenesis and adipogenesis of BMMSCs were evaluated the same as SCAP.

2.3. SCAP-Exo Isolation and Identification. SCAP-Exo were isolated according to previous protocols [17, 18]. For exosome isolation, a conventional culture medium was replaced with a serum-free medium when cells reached $60-80 \%$ confluence, and SCAP were cultured for an additional $48 \mathrm{~h}$. Then, the supernatant was centrifuged sequentially at $4^{\circ} \mathrm{C}$ $3,000 \times g$ for $20 \mathrm{~min}, 20,000 \times g$ for $30 \mathrm{~min}$, and $120,000 \times$ $g$ for $2 \mathrm{~h}$. Finally, the exosome pellets were resuspended in $200 \mu \mathrm{L}$ of PBS. $20 \mu \mathrm{L}$ SCAP-Exo was added to $30 \mu \mathrm{L}$ lysis buffer (Beyotime Biotech Co., Shanghai, China) for $1 \mathrm{~h}$ on ice. The total concentration of exosomes was detected by the bicinchoninic acid (BCA) kit (Beyotime, China). SCAPExo was stored at $-80^{\circ} \mathrm{C}$ for subsequent experiments.

The morphology of SCAP-Exo was identified with a transmission electron microscope (H-800, Hitachi, Japan). The size of these exosomes was analysed by nanoparticle tracking analysis. Furthermore, CD9 and Alix were detected by western blot using specific antibodies against CD9 (1:250, Abcam, USA) and Alix (1:500, Abcam, USA).

2.4. Preparation of Tooth Fragments. The teeth were obtained from clinically healthy premolars extracted for orthodontic reasons. The periodontal tissue of the root surface was removed with scalpel blades, and then, the cementum, dental pulp tissue, predentine, and partial dentine were removed with a fissure bur. The diameter of root canal space was expanded to about $3 \mathrm{~mm}$, and the teeth were split into $5 \mathrm{~mm}$ length root segments. Next, the tooth fragments were immersed in $17 \%$ EDTA for $5 \mathrm{~min}$, washed in deionised water for $10 \mathrm{~min}$ in an ultrasonic cleaner, and then immersed in 5\% EDTA for $10 \mathrm{~min}$, which was followed by washing in deionised water for $10 \mathrm{~min}$ in an ultrasonic cleaner. One of the tooth fragment orifices was sealed with glass ionomer cement (Fuji IX, GC, Tokyo, Japan). Then, it was stored in phosphate-buffered solution supplemented with $50 \mathrm{U} / \mathrm{mL}$ penicillin and $50 \mathrm{mg} / \mathrm{mL}$ streptomycin at $4^{\circ} \mathrm{C}$. 
2.5. Animal Experiments. This study was approved by the Ethics Committee of China Medical University for animal experiments (201315). All operations were performed on 6week-old nude mice $(n=10)$ under general anaesthesia. First, $50 \mu \mathrm{g} / \mathrm{mL}$ SCAP-Exo and a $4 \times 10^{5}$ BMMSCs with gelatine sponge (Xiang En, Jiang Xi, China) were introduced into the tooth fragments and transplanted subcutaneously into the backs of immunodeficient mice; $4 \times 10^{5}$ BMMSCs with gelatine sponge were inserted into the tooth fragments as control. Each mouse received four tooth fragments, two on each side. There were 10 samples in each group. Twelve weeks after transplantation, the mice were euthanised and the tooth fragments were taken out for histological analysis.

2.6. Histological Examination. Samples were fixed in 4\% paraformaldehyde for $24 \mathrm{~h}$ and decalcified in 10\% EDTA (pH 7.4, MeilunBio, China) for 3 months, and then, all samples were embedded in paraffin and cut into $5 \mu \mathrm{m}$ sections. For histological analysis, sections were stained with H\&E solution. Next, we counted the number of odontoblasts and measured the thickness of the new dentine using ImageJ software (1.50i, National Institutes of Health, Bethesda, MD, USA).

2.7. Exosomes Uptake Assay. SCAP-Exo were labelled using a PKH-26 Red Fluorescent Cell Linker Kit (Sigma, USA), as per the manufacturer's recommended protocol. Briefly, SCAP-Exo $(300 \mu \mathrm{g}$ protein equivalent of exosomes in $250 \mu \mathrm{L}$ Diluent $\mathrm{C})$ were stained with $\mathrm{PKH} 26(1 \mu \mathrm{L}$ of the PKH26 ethanolic dye solution in $250 \mu \mathrm{L}$ Diluent C) for $4 \mathrm{~min}$ at room temperature by pipetting. Then, $500 \mu \mathrm{L}$ of exosome-depleted serum was added to terminate the labelling reaction. Next, $2 \times 10^{4}$ BMMSCs were seeded on glass coverslips and placed inside a 12-well plate. Twenty-four hours after seeding, $\mathrm{PKH}-26$-labelled SCAP-Exo were incubated with BMMSCs for $3 \mathrm{~h}$. An equal volume of PBS was added to the control group. Cells were fixed with $4 \%$ paraformaldehyde, and the nuclei were stained with DAPI. Finally, we determined whether SCAP-Exo were internalised by BMMSCs using a fluorescent microscope.

2.8. Cell Counting Kit-8 (CCK-8) Assay. To assess the effect of SCAP-Exo on cell proliferation, BMMSCs were incubated with a medium supplemented with different concentrations of SCAP-Exo and proliferation was determined using a CCK-8 kit (Dojindo, Kumamoto, Japan). In brief, BMMSCs were seeded in 96-well plates (2,000 cells/well) and cultured with a conditioned medium (containing $0,5,20$, and $50 \mu \mathrm{g} / \mathrm{mL}$ SCAP-Exo). After 1, 3, and 5 days, approximately $10 \mu \mathrm{L}$ of CCK- 8 solution with $90 \mu \mathrm{L}$ of $\alpha$-MEM was added to each well, and the plate was incubated for another $2 \mathrm{~h}$ at $37^{\circ} \mathrm{C}$. The optical density was detected with a microplate reader (Tecan, Austria) at the wavelength of $450 \mathrm{~nm}$.

2.9. Ki-67 Staining Assay. BMMSCs $\left(2 \times 10^{4} /\right.$ well $)$ were seeded on glass coverslips placed inside a 12 -well plate and cultured for $48 \mathrm{~h}$. Cells were fixed with $4 \%$ paraformaldehyde and permeabilised using $0.3 \%$ triton $\mathrm{X}-100$ (Beyotime, China). Then, glass coverslips were incubated with an antiKi-67 (CST, USA) primary antibody at $4^{\circ} \mathrm{C}$ overnight. Next, samples were incubated with the fluorescent secondary anti- body (Proteintech, Chicago, USA) at room temperature for $2 \mathrm{~h}$; DAPI staining was then performed, and antiquench sealed tablets were used. Ki-67-positive and total cell numbers were counted based on 10 images per sample using a fluorescence microscope. The number of Ki-67-positive cells was indicated as a percentage of the total cell number.

2.10. In Vitro Osteo-/Odontogenic Differentiation Assay. BMMSCs $\left(1 \times 10^{5}\right.$ cells/well $)$ were seeded in 6-well plates and pretreated with a conditioned medium containing different doses of SCAP-Exo $(0,5,20$, and $50 \mu \mathrm{g} / \mathrm{mL})$ for 4 days. Then, culture medium was replaced with an osteo-/dentinogenic induction medium containing $1.8 \mathrm{mM}$ monopotassium phosphate (Sigma-Aldrich) and $10 \mathrm{nM}$ dexamethasone (Sigma-Aldrich). After incubation for 7 days, total mRNA was extracted, and 10 days later, proteins were isolated to assess the expression level of an osteogenic/dentinogenic marker.

2.11. Real-Time Polymerase Chain Reaction (PCR) Analysis. Real-time PCR was performed 30 cycles using the GoTaq ${ }^{\circledR}$ qPCR Master Mix (Promega Corporation, Madison, WI, USA) in a 7500 real-time PCR system (Applied Biosystems). The mRNA expression levels of dentin sialophosphoprotein $(D S P P)$, alkaline phosphatase $(A L P)$, and runt-related transcription factor 2 (Runx2) were evaluated. Glyceraldehyde3-phosphate dehydrogenase (GAPDH) was used as a housekeeping gene for normalisation. The data were calculated and statistically analysed using the formula $2^{-\Delta \Delta \mathrm{Ct}}$. The sequences of primers were as follows: DSPP forward primer, $5^{\prime}$-CTGTTGGGAAGAGCCAAGATAAG- ${ }^{\prime}$; DSPP reverse primer, $5^{\prime}$-CCAAGATCATTCCATGTTGTCCT- ${ }^{\prime} ;$ ALP forward primer, $5^{\prime}$-TAAGGACATCGCCTACCAGCTC-3'; ALP reverse primer, $5^{\prime}$-TCTTCCAGGTGTCAACGAGGT3'; Runx2 forward primer, 5'-GCACCCAGCCCATAAT AGA-3'; Run $x 2$ reverse primer, $5^{\prime}$-TTGGAGCAAGGAGA ACCC- $3^{\prime}$; GAPDH forward primer, $5^{\prime}$-CCGGCGTCCGA CCTGTGAAC-3'; GAPDH reverse primer, $5^{\prime}$-GGGCGA AGGCTCCAGAGGA-3'.

2.12. Western Blot Analysis. Total protein was extracted using lysis buffer (Beyotime Biotech Co., Shanghai, China). $20 \mu \mathrm{g}$ of whole protein was loaded and separated by $12 \%$ SDSpolyacrylamide gel electrophoresis and transferred electrophoretically to a polyvinylidene difluoride membrane (Millipore Corporation, USA). The membrane was blocked by incubation in $4 \%$ BSA for $1 \mathrm{~h}$ at room temperature and then incubated with anti-DSPP $(1: 500$, Santa Cruz Biotechnology, USA), anti-Runx2 (1:250, Santa Cruz Biotechnology, USA), anti-ALP (1:200, Santa Cruz Biotechnology), or anti- $\beta$-actin (1:1,000, Santa Cruz Biotechnology) primary antibodies at $4^{\circ} \mathrm{C}$ overnight. Goat anti-rabbit/anti-mouse IgG IRDyel $800 \mathrm{cw}$ secondary antibody $(1: 1,000$, Abbkine, USA) was used to incubate the membranes for $1 \mathrm{~h}$ at room temperature. The protein bands were detected with an Odyssey CLx instrument (LI-COR, Lincoln, NE, USA), and grayscale analysis was performed with ImageJ software (1.50i, National Institutes of Health, Bethesda, MA, USA). 


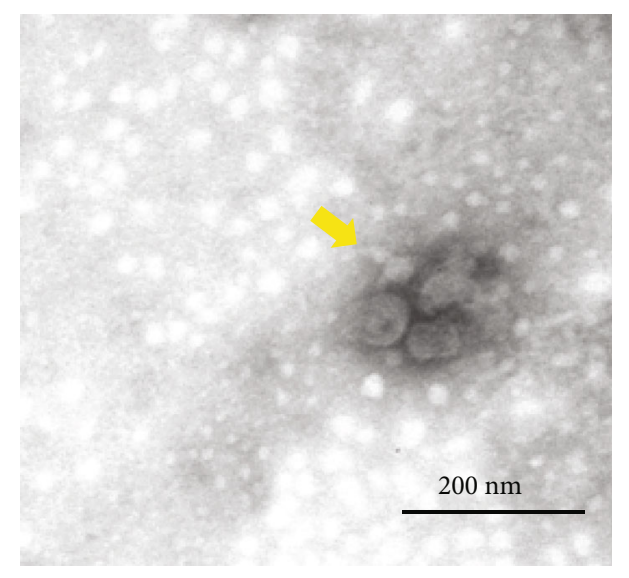

(a)

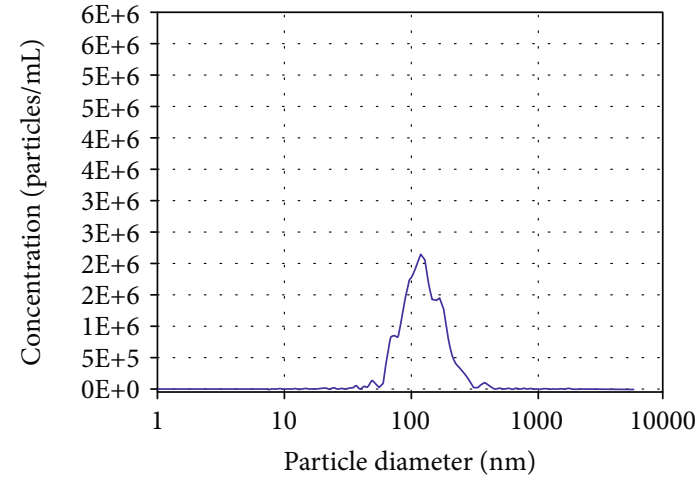

(b)

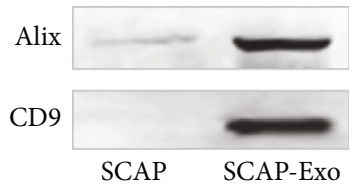

(c)

FIGURE 1: Identification of exosomes from stem cells of the apical papilla (SCAP-Exo). (a) Morphology of SCAP-Exo (yellow arrow) based on transmission electron microscopy. (b) Size distribution of particles in the pellet as measured by nanoparticle tracking analysis. (c) Western blot analysis showing that SCAP-Exo were positive for the exosomal-specific markers CD9 and Alix.

2.13. Alizarin Red S Staining. After culturing with an osteo/odontogenic induction medium for 3 weeks, all the cultures were fixed with $60 \%$ isopropanol for 1 min. Alizarin red S solution was added to each well for staining. Nonspecific staining was removed by repeated washing with distilled water. Semiquantitative analysis of mineralised nodule formation was performed with ImageJ software.

2.14. Statistical Analysis. Statistical analysis was performed using SPSS 20.0 software (SPSS Inc., Chicago, IL, USA). All data were recorded as the mean \pm SD and replicated for three independent experiments. The differences were tested by one-way analysis of variance (ANOVA). The differences were considered significant if the $P$ value was less than 0.05 .

\section{Results}

3.1. Identification of SCAP, BMMSCs, and SCAP-Exo. The majority of isolated SCAP retained a spindle shape and formed colonies in primary culture (Fig. S1A). When SCAP were cultured in an osteogenic- and adipogenicconditioned medium for 4 weeks, SCAP were found to form mineralised nodules based on Alizarin red $\mathrm{S}$ staining (Fig. S1B) and lipid droplets based on staining with Oil red O (Fig. S1C). Moreover, flow cytometric analysis showed that SCAP expressed mesenchymal stem cell surface markers including CD29, CD44, CD105, and CD146 but failed to express the haematopoietic markers CD34 and CD45 (Fig. S1D).

When BMMSCs were cultured for 7 days, cell adherent growth was observed by the microscope, showing the short spindle or polygon shape (Fig. S2A). After cultured with an osteogenic- or adipogenic-conditioned medium for 3 weeks, BMMSCs were also found to form mineralised nodules and lipid droplets (Fig. S2B, 2C).

By transmission electron microscopy, SCAP-Exo were observed to contain a bilayer membrane and cup-plateshaped structures (Figure 1(a)). Moreover, nanoparticle tracking analysis showed a major peak in particle size at $120.6 \mathrm{~nm}$ (Figure 1(b)). Furthermore, SCAP-Exo expressed the specific exosomal markers CD9 and Alix (Figure 1(c)) based on western blot.

\subsection{SCAP-Exo Promoted BMMSC-Based Dentine-Pulp} Complex Regeneration. As shown in the schematic diagram (Figure 2(a)), tooth fragments with SCAP-Exo, BMMSCs, and scaffolds were implanted subcutaneously into immunodeficient mice, whereas the control group was treated with the same preparation without SCAP-Exo. After 12 weeks, histological analysis showed that a new continuous dentine layer was formed in the SCAP-Exo group, in which the number of odontoblasts (yellow arrows) was significantly increased, with a high columnar shape and polarised morphology. They were located at the junction of pulp and predentine in an ordered arrangement, forming an odontoblast process into the dentinal tubules. In addition, more vascular lumens (red arrow) were also observed. In the control group, we did not observe the formation of this new dentine and odontoblast layer (Figure 2(b)). Both the thickness of the new dentine and the number of odontoblasts were higher in the SCAP-Exo group than that in the control group (Figures 2(c) and 2(d)). These data 


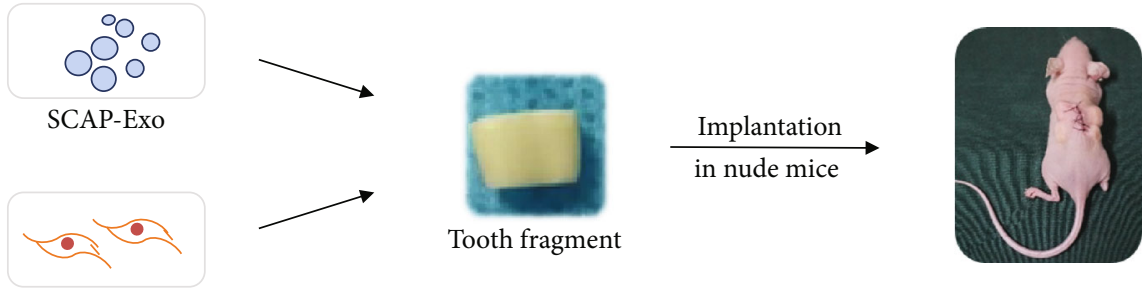

BMMSCs

(a)
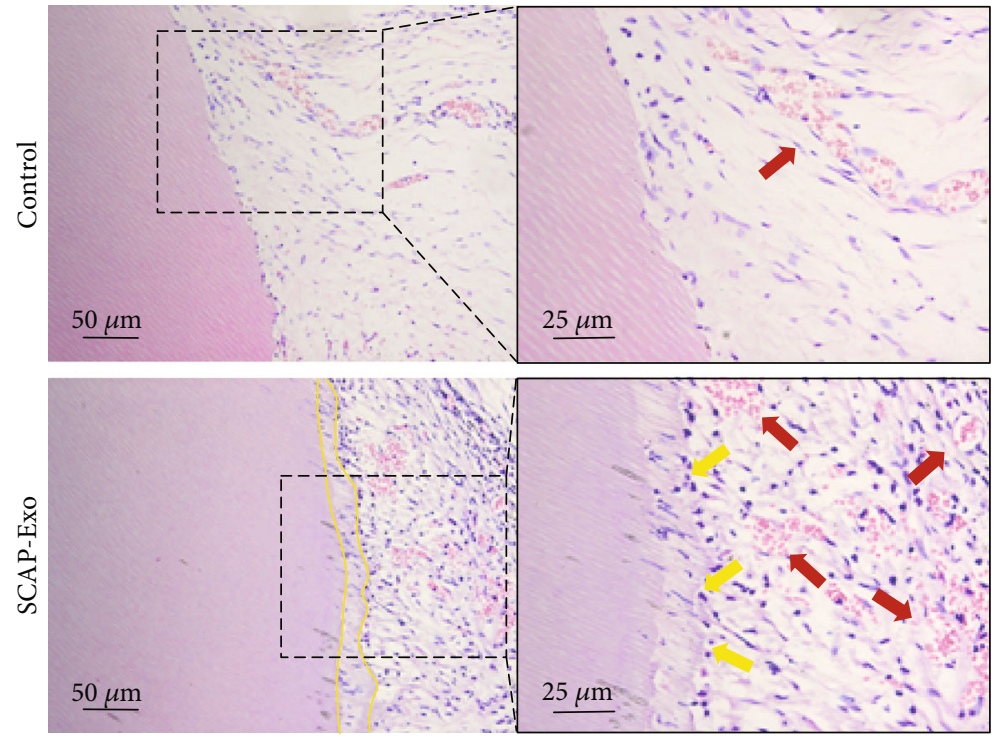

(b)

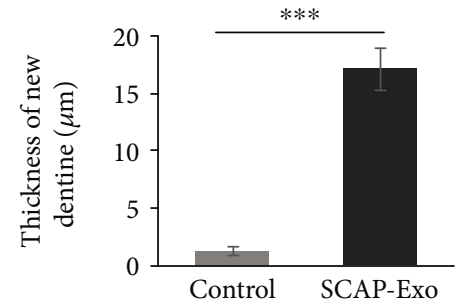

(c)

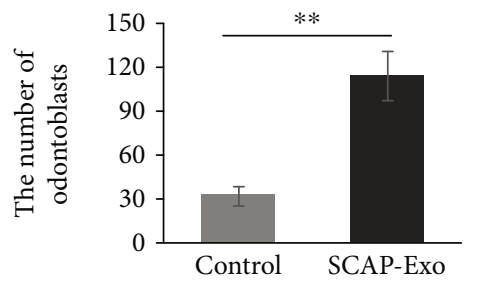

(d)

FIgURE 2: Exosomes from the stem cells of the apical papilla (SCAP-Exo) promoted the regeneration of the dentine-pulp complex in vivo. (a) Schematic diagram of the animal experiment: SCAP-Exo, bone marrow mesenchymal stem cells (BMMSCs), and gelatine sponges as scaffolds were inserted into the canal of the tooth fragments, while the control group was treated with the same preparation without SCAP-Exo. The tooth fragments were then implanted into the nude mice. (b) HE staining showed a newly continuous layer of dentine (dotted line), odontoblast-like cells with overt polarised morphology (yellow arrow), and enhanced vascular formation (red arrow) in the experimental group. (c, d) The thickness of the new dentine and the number of odontoblasts were higher in the SCAP-Exo group than that in the control group $\left.{ }^{* *} P<0.01,{ }^{* * *} P<0.001, n=10\right)$. Error bars indicate means \pm SD.

indicated that SCAP-Exo promoted BMMSC-based dentinepulp complex regeneration.

3.3. SCAP-Exo Were Endocytosed by BMMSCs. We next added PKH-26-labelled SCAP-Exo into the culture media of BMMSCs in vitro. After $3 \mathrm{~h}$, fluorescent staining showed that many SCAP-Exo (red) were located in the cytoplasm of DAPI-labelled BMMSCs (blue); however, only the nuclei of BMMSCs were stained with DAPI (blue) in the control group (Figure 3). These data suggested that BMMSCs could take up SCAP-Exo via endocytosis.
3.4. SCAP-Exo Induced the Dentinogenesis of BMMSCs. CCK-8 assays and Ki-67 staining showed that SCAP-Exo exerted no significant effects on the proliferation rate of BMMSCs (Figures 4(a) and 4(b)). To further explore whether and how SCAP-Exo regulate the osteo-/odontogenic differentiation of BMMSCs, we used SCAP-Exo to treat BMMSCs and then cultured cells under osteogenic induction conditions. Alizarin red S staining showed that mineralised nodule formation was increased markedly with SCAP-Exo treatment in a concentration-dependent manner (Figure 4(c)). Furthermore, we examined the expression 

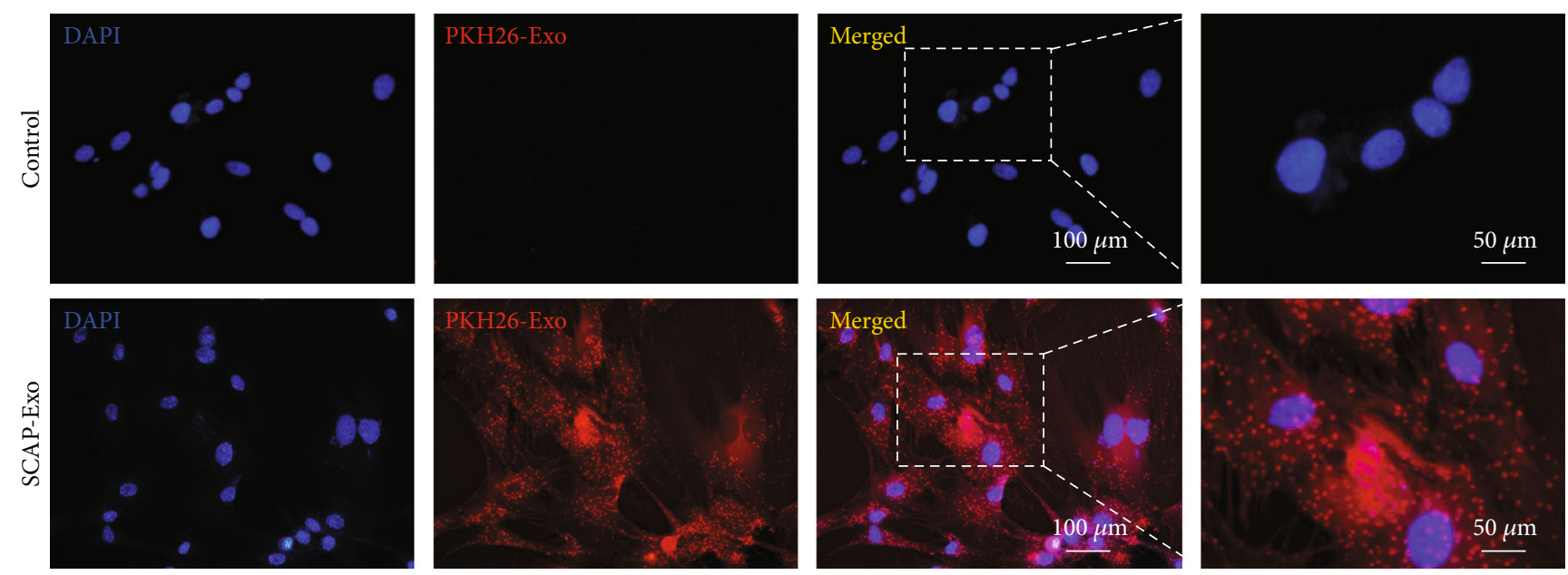

Figure 3: Exosomes from the stem cells of the apical papilla (SCAP-Exo) were endocytosed by bone marrow mesenchymal stem cells (BMMSCs). PKH-26-labelled SCAP-Exo (red) was internalised into the cytoplasm of DAPI-labelled BMMSCs (blue). In the negative control group of BMMSCs without exosomes, only the nuclei of BMMSCs were stained with DAPI (blue).

levels of osteo-/odontogenic genes and proteins. As shown in Figure 4(d), SCAP-Exo increased the gene expression of the dentinogenic marker DSPP, and the 20 and $50 \mu \mathrm{g} / \mathrm{mL}$ SCAP-Exo groups exhibited a more robust increase compared to that in the control group $(P<0.001)$. Nevertheless, SCAP-Exo had no significant effects on the gene expression levels of $A L P$ and Runx2. Consistent with qPCR analysis, the protein expression of DSPP also increased obviously upon $20 \mu \mathrm{g} / \mathrm{mL}$ and $50 \mu \mathrm{g} / \mathrm{mL}$ SCAP-Exo treatment $(P<0.001)$; however, SCAP-Exo also did not affect protein levels of ALP and Runx2 ( $P>0.05$; Figure 4(e)). Therefore, these data indicated that SCAP-Exo promoted the specific dentinogenesis of BMMSCs.

\section{Discussion}

Strategies for dentine-pulp complex regeneration are conventionally categorised into cell-based and cell-free therapies. Cell-based therapies consist of transplanting exogenous stem cells, loaded onto scaffolds incorporated with signalling molecules, into the root canal system of the host to allow regeneration. The modes of MSC-based therapy in tissue regeneration consist of MSCs mobilised to the site and differentiated into functional cells to replace damaged cells [19]. In general, dental-derived MSCs are selected for transplantation into the root canal. Xuan et al. have reported that human deciduous DPSCs are able to regenerate whole dental pulp and form new dentine to promote root development [20], while SCAP is derived from the immature tissue of the apical papilla and is composed of more undifferentiated cells than dental pulp [21]. However, the clinical translational application of MSCs is limited by ethical issues, availability, stem cell isolation and storage, and professional technical skills, among other factors. Additionally, increasing evidence has indicated that most exogenous MSCs quickly disappear posttransplant [22]. Based on "cell homing" theory, the cell-free approach achieves tissue regeneration by modulating the migration, proliferation, and differentiation of endogenous stem cells residing around the root apex. It is believed that "cell homing" is the basis for the majority of current clinical approaches to REPs [23]. Some scholars have suggested that cell niches for stem cell populations could establish a suitable microenvironment by producing immunoregulatory factors and promoting paracrine activities to stimulate the differentiation of progenitor cells in situ [19, 24, 25]. Natural compounds or bioactive substances have been shown to have the potential to induce specific dentinogenesis $[26,27]$.

Currently, the approach based on MSC-Exo provides new therapeutic alternatives for tissue regeneration. It has been shown that exosome characteristics differ in diverse cell types [28]. Further, the complex composition of MSC-Exo might mirror that of their parental cells and their ability to migrate towards specific tissue [22]. MSC-Exo also contain numerous bioactive molecules that can be transferred to target cells to influence their fate and tissue regeneration. A previous study indicated that exosomes isolated from dental pulp stem cells could induce the odontogenic differentiation in vitro and trigger the regeneration of dental pulp-like tissue in vivo [27]. In this study, we evaluated the potential of SCAP-Exo to promote dentine-pulp complex regeneration. Our in vivo study indicated that SCAP-Exo could facilitate the generation of the newly formed dentine tissues and odontoblasts. Furthermore, there were a large number of polar odontoblasts in an ordered arrangement. Odontoblast polarisation marks the morphological change from symmetrical mesenchymal cell to asymmetrical odontoblast, which is a prerequisite and fundamental step for tooth development and dentine formation [29]. In addition, we observed numerous blood vessels in newly formed pulp-dentine complex induced by SCAP-Exo in the root canal. Angiogenesis is a prerequisite for tissue regeneration through the provision of sufficient oxygen and nutrients for tissue survival [30]. It has been reported that dental pulp stem cell-derived exosomes had an effect on angiogenesis [31]. SCAP can secrete a large number of proangiogenic factors [32]. There is a need to clarify whether SCAP-Exo can promote angiogenesis or not and its related mechanism in the future. Summarily, the 


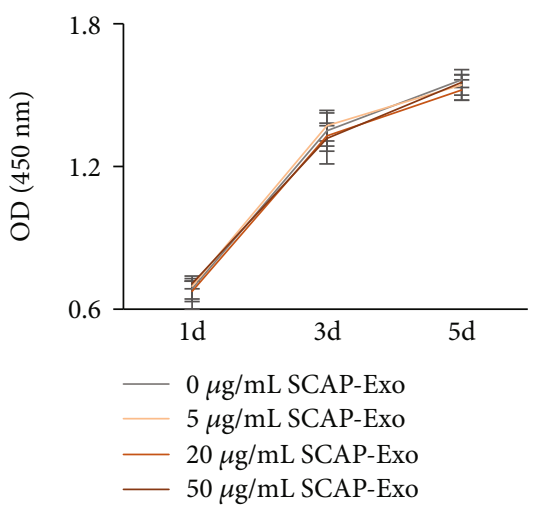

(a)

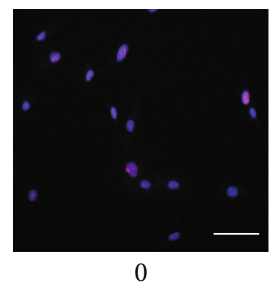

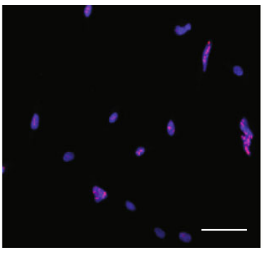

5

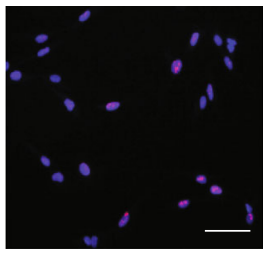

20

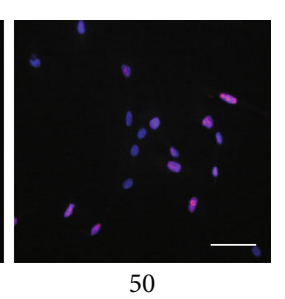

50

(b)
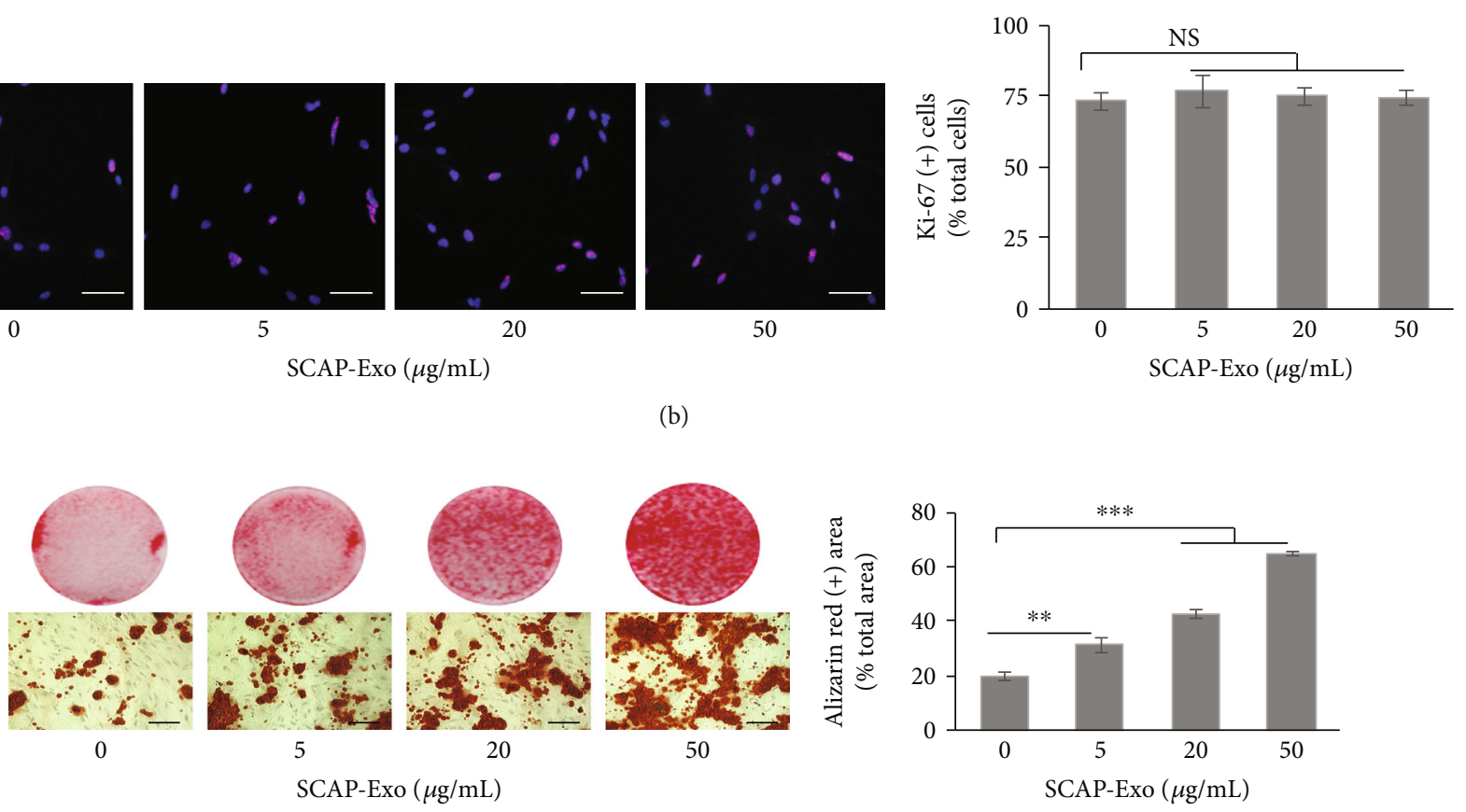

(c)
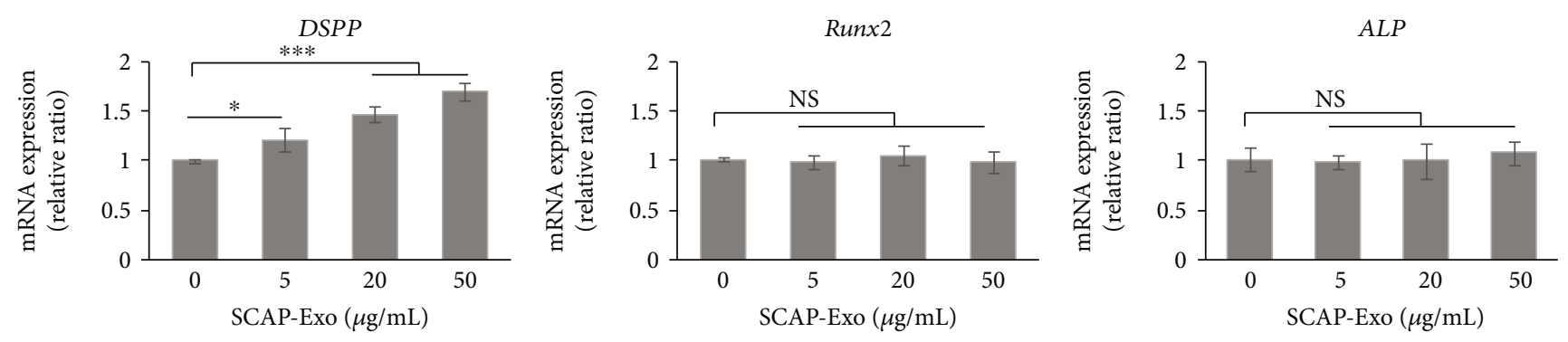

(d)

Figure 4: Continued. 


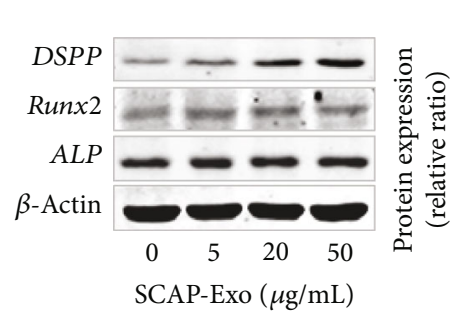

SCAP-Exo $(\mu \mathrm{g} / \mathrm{mL})$
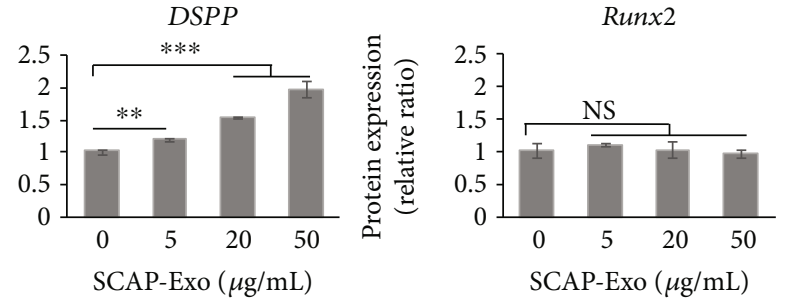

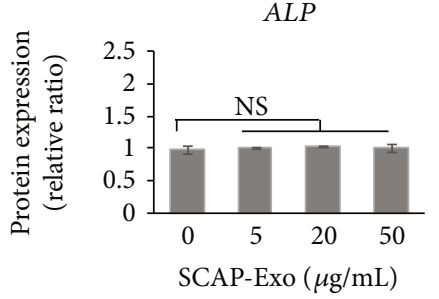

(e)

FIGURE 4: Exosomes from stem cells of the apical papilla (SCAP-Exo) induced the odontogenic differentiation of bone marrow mesenchymal stem cells (BMMSCs). (a, b) CCK-8 and Ki-67 assays showed that SCAP-Exo had no significant effect on the proliferation of BMMSCs. Scale bars $=100 \mu \mathrm{m}$. (c) Alizarin red S staining showed that SCAP-Exo could increase mineralised nodule formation in a dose-dependent manner. Scale bars $=100 \mu \mathrm{m}$. (d) Real-time PCR analysis revealed that SCAP-Exo could augment DSPP mRNA expression levels in BMMSCs without exerting effects on Runx2 and ALP mRNA expression levels. (e) Western blot analysis indicated that the protein expression levels of ALP and Runx2 were not significantly changed, but that high doses (20 and $50 \mu \mathrm{g} / \mathrm{mL})$ of SCAP-Exo significantly improved the protein expression levels of DSPP $\left({ }^{*} P<0.05,{ }^{* *} P<0.01\right.$, and ${ }^{* * *} P<0.001$, NS $=$ not significant, $\left.n=3\right)$. Error bars indicate means \pm SD.

histologic findings suggested that SCAP-Exo could improve the dentine-pulp complex regeneration in REPs.

To explore the underlying mechanisms through which SCAP-Exo promote the regeneration of pulp-dentine complex, we evaluated their effects on the proliferation and differentiation of BMMSCs in vitro. We found that SCAP-Exo did not affect BMMSC proliferation but significantly regulated their dentinogenesis by enhancing DSPP expression in BMMSCs, as well as the formation of mineralised nodules. DSPP, a precursor protein, can be cleaved into two proteins, specifically dentine sialoprotein (DSP) and dentine phosphoprotein (DPP), which are believed to play a crucial role in dentine formation [33]. DSPP is secreted by odontoblasts and is the most abundant in the extracellular matrix; moreover, it can serve as a template for mineral nucleation and growth to mediate the mineralisation of extracellular matrix during dentine formation [34]. In addition, it is a terminal differentiation marker of odontoblasts [35]. Recently, Frozoni used BMMSCs in pulp cap in a mouse model and they found that GFP labelled-BMMSCs were directly utilised and GFP-labelled DSP positive odontoblasts were observed in the root canal 7 weeks later [36]. This study may shed some light on how to explain their observations. The endogenous dental pulp stem cells around the pulp exposure site might transfer specific substances into the transplanted BMMSCs and induce dentinogenesis of BMMSCs via a paracrine effect. In particular, in this study, SCAP-Exo treatment did not significantly influence the expression levels of another two mineralisation-related proteins, ALP and Runx2. We speculated that SCAP-Exo might mainly promote the secretion of DSPP rather than the differentiation of BMMSCs.

At present, how exosomes influence recipient cells remain elusive. Exosomes contain various RNAs (including mRNA, miRNA, and tRNA) and proteins [10, 37]. Baglio et al. showed that the five most abundant miRNAs in MSCExo account for a high proportion (50\%) of the total miRNA, which indicates that specific miRNAs present in large amounts might have a physiological impact on target cells [38]. In addition, exosomal proteins could be directly utilised by recipient cells. Our previous study showed that recipient
MSCs can receive exosomes from donor MSCs and reuse Fas derived from these exosomes to improve recipient MSC function [39]. In this study, we considered that SCAP-Exo might transfer the biological information of periapical tissue to reprogram the function of BMMSCs. However, the detailed mechanisms through which SCAP-Exo influence the biological function of BMMSCs need to be further elucidated.

SCAP-Exo serve as systemic cell-cell communication mediators that play an important role in tissue regeneration. We believed that exosomes released by various cell types in different states might contain diverse contents to deliver specific information, which could have different implications for the target cells. The application of SCAP-Exo could thus provide a new strategy to promote dentine-pulp complex regeneration after REPs. Further studies will need to focus on establishing an animal model of periapical periodontitis in permanent teeth with open apices to determine the efficacy of SCAP-Exo in REPs. However, it is crucial to select a suitable scaffold for carrying exosomes to the root canal. There is evidence that the geometrical and mechanical properties of scaffolds can influence cell behaviour and the adhesion of MSCs to the scaffold surfaces [40-42]. Further studies are needed to select suitable scaffold materials for future use of SCAP-Exo in the root canal and to elucidate the possible interaction between SCAP-Exo and scaffold materials during REPs as well.

\section{Conclusions}

SCAP-Exo could be endocytosed by BMMSCs, obviously improved their specific dentinogenesis, and promoted dentine-pulp complex regeneration. This study provided an optimised method for cell homing-based REPs in the clinic. It could be a promising therapeutic approach that uses standardised exosomal reagents in regenerative endodontics.

\section{Data Availability}

The data used to support the findings of this study are available from the corresponding author upon request. 


\section{Conflicts of Interest}

The authors have stated explicitly that there is no conflict of interest in connection with this article.

\section{Acknowledgments}

This work was supported by grants from the National Natural Science Foundation of China (no. 81771059), the Natural Science Foundation of Liaoning Province (no. 201602842), and Youth Research Fund of China Medical University (grant no. QGZD2018083). Some work was supported by the Key Laboratory of Immunodermatology (China Medical University), Ministry of Education.

\section{Supplementary Materials}

Supplementary Figure 1: identification of stem cells of apical papilla (SCAP). (A) SCAP formed colony-forming units in primary culture. (B) Alizarin red S staining showed the formation of mineralised nodules. (C) Oil red $\mathrm{O}$ staining showed lipid droplet formation. (D) Flow cytometric analysis showed that SCAP were positive for CD29, CD44, CD105, and CD146 but negative for CD34 and CD45. Supplementary Figure 2: identification of bone marrow mesenchymal stem cells (BMMSCs). (A) The morphology of BMMSCs. (B) Alizarin red $\mathrm{S}$ staining showed the formation of mineralised nodules. (C) Oil red O staining showed lipid droplet formation. (Supplementary Materials)

\section{References}

[1] M. Nakashima and A. Akamine, "The application of tissue engineering to regeneration of pulp and dentin in endodontics," Journal of Endodontics, vol. 31, no. 10, pp. 711-718, 2005.

[2] B. N. Lee, J. W. Moon, H. S. Chang, I. N. Hwang, W. M. Oh, and Y. C. Hwang, "A review of the regenerative endodontic treatment procedure," Restorative Dentistry \& Endodontics, vol. 40, no. 3, pp. 179-187, 2015.

[3] S. G. Kim, M. Malek, A. Sigurdsson, L. M. Lin, and B. Kahler, "Regenerative endodontics: a comprehensive review," International Endodontic Journal, vol. 51, no. 12, pp. 1367-1388, 2018.

[4] D. D. Zhang, X. Chen, Z. F. Bao, M. Chen, Z. J. Ding, and M. Zhong, "Histologic comparison between platelet-rich plasma and blood clot in regenerative endodontic treatment: an animal study," Journal of Endodontics, vol. 40, no. 9, pp. 1388-1393, 2014.

[5] P. Becerra, D. Ricucci, S. Loghin, J. L. Gibbs, and L. M. Lin, "Histologic study of a human immature permanent premolar with chronic apical abscess after revascularization/revitalization," Journal of Endodontics, vol. 40, no. 1, pp. 133-139, 2014.

[6] L. Lei, Y. Chen, R. Zhou, X. Huang, and Z. Cai, "Histologic and immunohistochemical findings of a human immature permanent tooth with apical periodontitis after regenerative endodontic treatment," Journal of Endodontics, vol. 41, no. 7, pp. 1172-1179, 2015.

[7] P. J. Palma, J. C. Ramos, J. B. Martins et al., "Histologic evaluation of regenerative endodontic procedures with the use of chitosan scaffolds in immature dog teeth with apical periodon- titis," Journal of Endodontics, vol. 43, no. 8, pp. 1279-1287, 2017.

[8] L. da Silva Meirelles, A. M. Fontes, D. T. Covas, and A. I. Caplan, "Mechanisms involved in the therapeutic properties of mesenchymal stem cells," Cytokine \& Growth Factor Reviews, vol. 20, no. 5-6, pp. 419-427, 2009.

[9] D. G. Phinney, M. di Giuseppe, J. Njah et al., "Mesenchymal stem cells use extracellular vesicles to outsource mitophagy and shuttle microRNAs," Nature Communications, vol. 6, no. 1, article 8472, 2015.

[10] A. Marote, F. G. Teixeira, B. Mendes-Pinheiro, and A. J. Salgado, "MSCs-derived exosomes: cell-secreted nanovesicles with regenerative potential," Frontiers in Pharmacology, vol. 7, p. 231, 2016.

[11] B. Yu, X. Zhang, and X. Li, "Exosomes derived from mesenchymal stem cells," International Journal of Molecular Sciences, vol. 15, no. 3, pp. 4142-4157, 2014.

[12] S. Raik, A. Kumar, and S. Bhattacharyya, "Insights into cellfree therapeutic approach: role of stem cell "soup-ernatant"," Biotechnology and Applied Biochemistry, vol. 65, no. 2, pp. 104-118, 2018.

[13] Y. Nakamura, S. Miyaki, H. Ishitobi et al., "Mesenchymalstem-cell-derived exosomes accelerate skeletal muscle regeneration," FEBS Letters, vol. 589, no. 11, pp. 1257-1265, 2015.

[14] L. Shao, Y. Zhang, B. Lan et al., "MiRNA-sequence indicates that mesenchymal stem cells and exosomes have similar mechanism to enhance cardiac repair," Bio Med Research International, vol. 2017, article 4150705, pp. 1-9, 2017.

[15] H. G. Zhang and W. E. Grizzle, "Exosomes: a novel pathway of local and distant intercellular communication that facilitate the growth and metastasis of neopastic lesion," The American Journal of Pathology, vol. 184, no. 1, pp. 28-41, 2014.

[16] K. C. Lin, H. K. Yip, P. L. Shao et al., "Combination of adiposederived mesenchymal stem cells (ADMSC) and ADMSCderived exosomes for protecting kidney from acute ischemiareperfusion injury," International Journal of Cardiology, vol. 216, pp. 173-185, 2016.

[17] X. Kou, X. Xu, C. Chen et al., "The Fas/Fap-1/Cav-1 complex regulates IL-1RA secretion in mesenchymal stem cells to accelerate wound healing," Science Translational Medicine, vol. 10, no. 432, article eaai8524, 2018.

[18] A. Wang, J. Liu, X. Zhuang et al., "Identification and comparison of piRNA expression profiles of exosomes derived from human stem cells from the apical papilla and bone marrow mesenchymal stem cells," Stem Cells and Development, vol. 29, no. 8, pp. 511-520, 2020.

[19] Y. Wang, X. Chen, W. Cao, and Y. Shi, "Plasticity of mesenchymal stem cells in immunomodulation: pathological and therapeutic implications," Nature Immunology, vol. 15, no. 11, pp. 1009-1016, 2014.

[20] K. Xuan, B. Li, H. Guo et al., "Deciduous autologous tooth stem cells regenerate dental pulp after implantation into injured teeth," Science Translational Medicine, vol. 10, no. 455, article eaaf3227, 2018.

[21] S. Cantore, A. Ballini, D. de Vito et al., "Characterization of human apical papilla-derived stem cells," Journal of Biological Regulators and Homeostatic Agents, vol. 31, no. 4, pp. 901-910, 2017.

[22] D. G. Phinney and M. F. Pittenger, "Concise review: MSCderived exosomes for cell-free therapy," Stem Cells, vol. 35, no. 4 , pp. 851-858, 2017. 
[23] S. Eramo, A. Natali, R. Pinna, and E. Milia, "Dental pulp regeneration via cell homing," International Endodontic Journal, vol. 51, no. 4, pp. 405-419, 2018.

[24] M. Boccellino, D. di Stasio, G. Dipalma et al., "Steroids and growth factors in oral squamous cell carcinoma: useful source of dental-derived stem cells to develop a steroidogenic model in new clinical strategies," European Review for Medical and Pharmacological Sciences, vol. 23, no. 20, pp. 8730-8740, 2019.

[25] A. Ballini, S. Scacco, D. Coletti, S. Pluchino, and M. Tatullo, "Mesenchymal stem cells as promoters, enhancers, and playmakers of the translational regenerative medicine," Stem Cells International, vol. 2017, Article ID 3292810, 2 pages, 2017.

[26] A. di Benedetto, F. Posa, S. de Maria et al., "Polydatin, natural precursor of resveratrol, promotes osteogenic differentiation of mesenchymal stem cells," International Journal of Medical Sciences, vol. 15, no. 9, pp. 944-952, 2018.

[27] C. C. Huang, R. Narayanan, S. Alapati, and S. Ravindran, "Exosomes as biomimetic tools for stem cell differentiation: applications in dental pulp tissue regeneration," Biomaterials, vol. 111, pp. 103-115, 2016.

[28] C. Charoenviriyakul, Y. Takahashi, M. Morishita, A. Matsumoto, M. Nishikawa, and Y. Takakura, "Cell typespecific and common characteristics of exosomes derived from mouse cell lines: yield, physicochemical properties, and pharmacokinetics," European Journal of Pharmaceutical Sciences, vol. 96, pp. 316-322, 2017.

[29] B. Chang, K. K. H. Svoboda, and X. H. Liu, "Cell polarization: from epithelial cells to odontoblasts," European Journal of Cell Biology, vol. 98, no. 1, pp. 1-11, 2019.

[30] P. Carmeliet, "Angiogenesis in life, disease and medicine," Nature, vol. 438, no. 7070, pp. 932-936, 2005.

[31] X. Xian, Q. Gong, C. Li, B. Guo, and H. Jiang, "Exosomes with highly angiogenic potential for possible use in pulp regeneration," Journal of Endodontics, vol. 44, no. 5, pp. 751-758, 2018.

[32] A. Bakopoulou, A. Kritis, D. Andreadis et al., "Angiogenic potential and secretome of human apical papilla mesenchymal stem cells in various stress microenvironments," Stem Cells and Development, vol. 24, no. 21, pp. 2496-2512, 2015.

[33] H. Ritchie, "The functional significance of dentin sialoproteinphosphophoryn and dentin sialoprotein," International Journal of Oral Science, vol. 10, no. 4, p. 31, 2018.

[34] Y. Yamakoshi, "Dentin sialophophoprotein (DSPP) and dentin," Journal of Oral Biosciences, vol. 50, no. 1, pp. 33-44, 2008.

[35] Y. Chen, Y. Zhang, A. Ramachandran, and A. George, "DSPP is essential for normal development of the dental-craniofacial complex," Journal of Dental Research, vol. 95, no. 3, pp. 302$310,2015$.

[36] M. Frozoni, M. R. Marques, S. K. Hamasaki, N. T. Mohara, A. de Jesus Soares, and A. A. Zaia, "Contribution of bone marrow-derived cells to reparative dentinogenesis using bone marrow transplantation model," Journal of Endodontics, vol. 46, no. 3, pp. 404-412, 2020.

[37] S. W. Ferguson, J. Wang, C. J. Lee et al., "The microRNA regulatory landscape of MSC-derived exosomes: a systems view," Scientific Reports, vol. 8, no. 1, p. 1419, 2018.

[38] S. R. Baglio, K. Rooijers, D. Koppers-Lalic et al., "Human bone marrow- and adipose-mesenchymal stem cells secrete exosomes enriched in distinctive miRNA and tRNA species," Stem Cell Research \& Therapy, vol. 6, no. 1, p. 127, 2015.
[39] S. Liu, D. Liu, C. Chen et al., "MSC transplantation improves osteopenia via epigenetic regulation of notch signaling in lupus," Cell Metabolism, vol. 22, no. 4, pp. 606-618, 2015.

[40] S. Bose, M. Roy, and A. Bandyopadhyay, "Recent advances in bone tissue engineering scaffolds," Trends in Biotechnology, vol. 30, no. 10, pp. 546-554, 2012.

[41] A. A. Zadpoor, "Bone tissue regeneration: the role of scaffold geometry," Biomaterials Science, vol. 3, no. 2, pp. 231-245, 2015.

[42] S. Cantore, V. Crincoli, A. Boccaccio et al., "Recent advances in endocrine, metabolic and immune disorders: mesenchymal stem cells (MSCs) and engineered scaffolds," Endocrine Metabolic \& Immune Disorders-Drug Targets, vol. 18, no. 5, pp. 466-469, 2018. 